\title{
Gaze Guidance for Improved Password Recollection
}

\author{
Srinivas Sridharan* Brendan John $\quad$ Darrel Pollard $\quad$ Reynold Bailey
}

Rochester Institute of Technology

\begin{abstract}
Most computer systems require user authentication, which has led to an increase in the number of passwords one has to remember. In this paper we explore if spatial visual cues can be used to improve password recollection. Specifically, we consider if associating each character in a password to user-defined spatial regions in an image facilitates better recollection. We conduct a user study where participants were asked to recall randomly generated numeric passwords under the following conditions: no image association (No-Image), image association (Image-Only), image association combined with overt visual cues (Overt-Guidance), and image association combined with subtle visual cues (Subtle-Guidance). We measured the accuracy of password recollection and response time as well as average dwell-time at target locations for the gaze guided conditions. Subjects performed significantly better on password recollection when they were actively guided to regions in the associated image using overt visual cues. Accuracy of password recollection using subtle cues was also higher than the No-Image and Image-Only conditions, but the effect was not significant. No significant difference was observed in the average dwell-times between the overt and subtle guidance approaches.
\end{abstract}

Keywords: gaze guidance, password recollection, eye-tracking

Concepts: •Security and privacy $\rightarrow$ Graphical / visual passwords; •Computing methodologies $\rightarrow$ Image manipulation;

\section{Introduction and Background}

Recalling passwords has become a routine activity of modern life. Security of user information is critical due to the rapid increase of user accessible computing devices and cloud-based application interfaces. Many systems mandate that users create complicated passwords which are required to be a certain length and must include a combination of alphanumeric and special characters. For additional security, certain systems also require periodic password changes and the new passwords cannot be similar to previously used passwords. Shorter numeric passwords are also used widely for ATMs, locker systems, and mobile devices. Remembering all of these passwords is a very challenging task. Furthermore, the procedures for recovering/resetting passwords are time consuming and often lead to user frustration. The use of password managing software can help address these challenges however such technologies are not yet widely adopted.

This paper explores if spatial visual cues can improve password recollection. Specifically we examine if associating each character

\footnotetext{
*e-mail: sxs9716@rit.edu
}

Permission to make digital or hard copies of all or part of this work for personal or classroom use is granted without fee provided that copies are not made or distributed for profit or commercial advantage and that copies bear this notice and the full citation on the first page. Copyrights for components of this work owned by others than ACM must be honored. Abstracting with credit is permitted. To copy otherwise, or republish, to post on servers or to redistribute to lists, requires prior specific permission and/or a fee. Request permissions from permissions@acm.org. (c) 2016 ACM.

ETRA 2016, March 14 - 17, 2016, Charleston, SC, USA

ISBN: 978-1-4503-4125-7/16/03

DOI: http://dx.doi.org/10.1145/2857491.2857537 of a password with a spatial region of an image and then guiding the viewer to revisit these regions leads to better recollection. Also known as the method of loci, this idea of associating items that one would like to remember with auxiliary spatial information has been shown to enhance memory recall [Hutton 1993]. Variations of this technique are commonly used by memory competition participants.

Researchers have proposed many theories related to pre-attentive visual processing of information from the environment. The scanpath theory [Privitera 2006] suggests that successive saccades and fixations made while viewing an image become part of the lasting memory and if replayed can lead to better scene recollection. Guided search theory [Wolfe 1994] models the goals of the viewer's search behavior and attempts to differentiate valid information from distractors. Researchers have also studied how image features influence visual attention and enable task completion [Itti and Koch 2000]. It has also been shown that visual short term memory is highly dependent on the regions in the scene that attract visual attention [Awh et al. 2000].

Guiding a viewer's attention to relevant regions of a scene has been shown to aid spatial learning, problem solving, and search task completion [Sridharan et al. 2012; Groen and Noyes 2010; McNamara et al. 2009; Qvarfordt et al. 2010]. Computer-based techniques for guiding attention can be classified as overt or subtle. Overt techniques involve the use of highly salient cues or permanent alterations to the image in order to highlight regions of interest. Subtle techniques on the other hand, rely on temporary or subdued changes in the imagery to guide visual attention. For example, subtle guidance can be achieved by adjusting the contrast, color, or luminance in order to increase or decrease the visual saliency of target regions [Veas et al. 2011; Lu et al. 2014]. In this paper, we use the subtle gaze direction (SGD) approach [Bailey et al. 2009] to guide viewer's attention about the scene. This technique uses brief image space modulations in the peripheral regions of the field of view in order to attract viewer attention. A fast eye tracker is used to monitor the viewer's gaze in real time and the modulations are turned off before the viewer can fixate on them.

We present the results of a user study where participants were asked to recall randomly generated 7-digit passwords under the following conditions: no image association (No-Image), image association (Image-Only), image association combined with overt visual cues (Overt-Guidance), and image association combined with subtle visual cues (Subtle-Guidance). We chose 7-digits based on Miller's observation [Miller 1956] that the average human can only hold $7 \pm 2$ items in working memory. We report on the accuracy of password recollection and response time for each of these conditions. Additionally we report on the average dwell-time at target locations for the gaze guided conditions.

\section{Experiment Design}

We tested subjects' ability to recall three randomly-generated numeric passwords under each of the following conditions:

- Condition 1: No-Image - during a memorization stage, participants were provided with three randomly generated passwords against a black background. In a subsequent recall stage participants were asked to recollect the three passwords. 


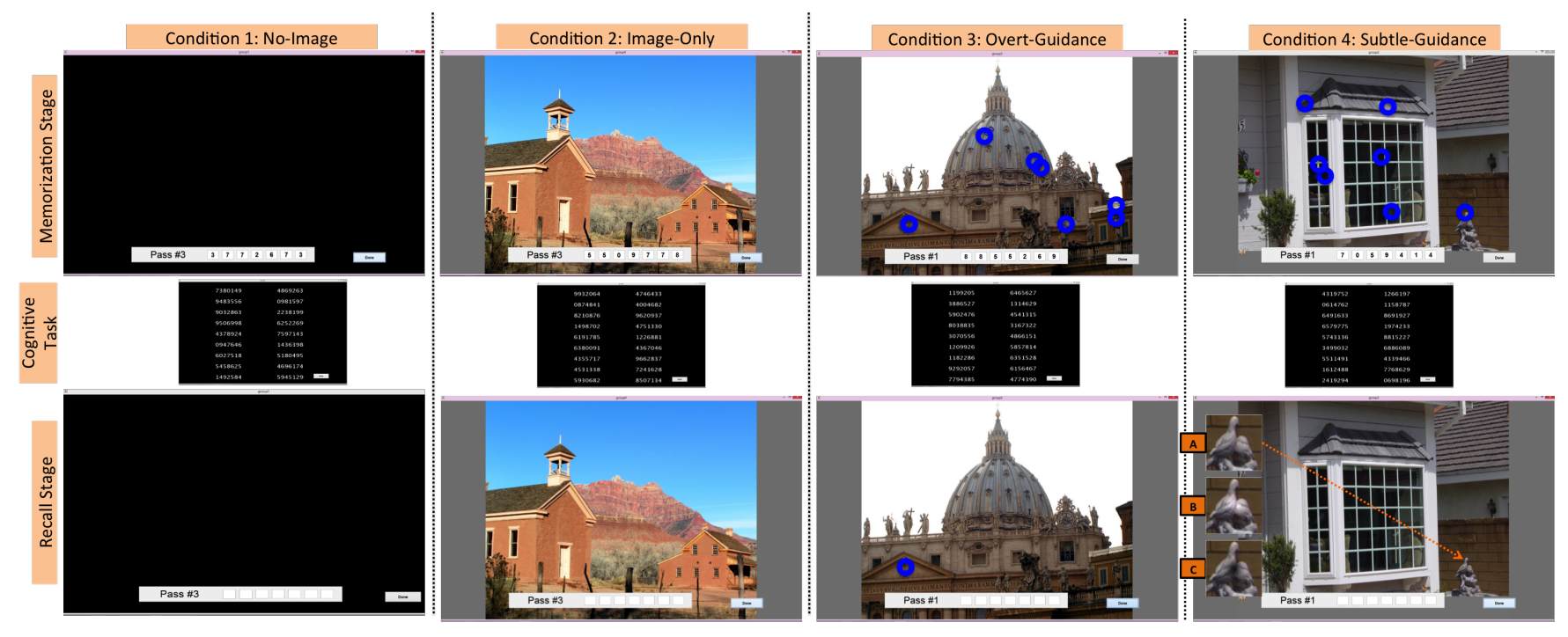

Figure 1: Experiment Design. Images in the top and bottom rows are sample screen shots from the memorization and recall stages respectively. The columns indicate the four conditions namely, No-Image, Image-Only, Overt-Guidance, and Subtle-Guidance. Each image in the middle row is a screen shot containing 18 random 7-digit numbers from the cognitive task stage. In the memorization stage, for conditions 3 and 4, participants associate each digit in the password to regions in the image using a mouse. The selected regions are indicated by blue circles. For condition 3, during the recall stage, the participants are overtly guided to the previously marked regions in sequence using blue circles. For condition 4, the participants are guided to the previously marked regions using subtle image space modulations presented only in the viewer's peripheral field of view. Inset image A shows a sub-image extracted around the password associated region selected by the participant. Inset images $B$ and $C$ show the bright and dark extents of the luminance modulations respectively.

- Condition 2: Image-Only - during a memorization stage, participants were provided with three randomly generated passwords along with a unique image for each password. In a subsequent recall stage, participants were shown the images and asked to recollect the corresponding passwords.

- Condition 3: Overt-Guidance - during a memorization stage, participants were provided with three randomly generated passwords along with a unique image for each password. Participants were asked to associate specific regions of the image with each digit in the password. In a subsequent recall stage, participants were shown the images and overtly guided to the marked regions in sequence and asked to recollect the corresponding passwords.

- Condition 4: Subtle-Guidance - during a memorization stage, participants were provided with three randomly generated passwords along with a unique image for each password. Participants were asked to associate specific regions of the image with each digit in the password. In a subsequent recall stage, participants were shown the images and subtly guided to the marked regions in sequence and asked to recollect the corresponding passwords.

Between the memorization and recall stages, the participants were presented with the cognitive task of reading 18 randomly generated 7-digit numbers out loud. Figure 1 illustrates each condition.

\subsection{Stimuli}

The stimuli images for all conditions were presented on a 22 inch monitor, operating at $60 \mathrm{~Hz}$ with a resolution of $1680 \mathrm{x}$ 1050. The images were obtained from the MIT benchmark images dataset [Judd et al. 2012]. Nine images (3 for each of the imagebased conditions) were chosen by the researchers. The images contained multiple objects and salient regions to facilitate password association. All images were padded with a gray background to fill the screen. A SensoMotoric Instruments iView X Remote Eye Tracking Device operated at $120 \mathrm{~Hz}$, with a manufacturer-reported gaze position accuracy $<0.5^{\circ}$, was used in this experiment. A chin-rest was not used in this experiment in order to encourage natural viewing. The participants were seated with a viewing distance of $\sim 70 \mathrm{~cm}$ from the eye tracker.

\subsection{Participants}

17 participants ( 4 females, 13 males), between the ages of 19 and 30 (avg. 24) volunteered to participate in this study. All participants reported normal and corrected-to-normal vision with no color vision abnormalities. Two participants' data were removed from analysis due to tracking loss.

\subsection{Procedure}

Each participant was asked to recall a total of 12 passwords, three for each condition. New passwords were generated for each subject. The order in which the conditions were presented to the subjects was randomized. There was a 2-minute break between each condition to allow the researchers time to modify the experiment parameters. The participants viewed a blank screen during this time.

\subsubsection{Memorization Stage}

During the memorization stage, three unique randomly generated 7-digit passwords were shown to the participant consecutively. The participants were given unlimited time to attempt to memorize the password and had to click on a GUI button to move to the next one. For the Overt-Guidance and Subtle-Guidance conditions, they were asked to first associate a region of the image with each of the 7 password digits. This was done by clicking on the 7 desired regions in order. The clicked regions were highlighted using a blue circle 
and remained highlighted during memorization. Participants were not eye-tracked during the memorization stage.

\subsubsection{Cognitive Task}

Following the memorization stage, 18 random seven digit numbers were displayed on screen. The participants were asked to read them aloud. This was done to increase the difficulty of the recollection task. After reading the numbers, the participant clicked a button to move to the next stage. Participants were not eye-tracked while reading.

\subsubsection{Recall Stage}

At the beginning of the recall stage, a 9-point eye-tracker calibration was performed, followed by a 4-point validation using manufacturer provided techniques. The validation step revealed calibration accuracy for all participants to be $<1^{\circ}$. The participants were asked to recall the 3 passwords from the memorization stage by entering them on three consecutive screens. For each screen, the participants were required to wait 25 seconds before entering their response. An audible beep was played after 25 seconds, and the keyboard and mouse were activated. The 25 second delay was necessary to allow for the active guidance of participants' gaze in conditions 3 and 4 . The delay was applied to all conditions for consistency. For the gaze guided conditions, the previously marked regions were highlighted in order and cycled through three times. For the Overt-Guidance condition, this involved displaying each of the marked regions using a blue circle for 1 -second (total 21 seconds). For the Subtle-Guidance condition, the timing is non-deterministic since modulations are dependent on the subject's gaze. Modulations are presented only in the peripheral regions of the field of view and are terminated once a saccade is detected towards the target. We rely on published results indicating that this termination criteria is met within 0.5 seconds for approximately 75 percent of target regions and within 1 second for approximately 90 percent of target regions [Bailey et al. 2009]. So the expectation is that $3 \mathrm{cy}-$ cles of the password modulations would be completed within 21 seconds. We added 4 seconds to further increase the likelihood of cycle completion.

For the Subtle-Guidance condition, modulations occurred at a frequency of $20 \mathrm{~Hz}$ and the intensity of the modulated pixels varied along a sinusoidal curve between $\pm 10 \%$ of the original pixel intensity value multiplied by the corresponding value from a predefined Gaussian distribution. In addition to gaze information, the participants' input password and response time after the beep were recorded.

\section{Results and Discussion}

\subsection{Accuracy}

The task of memorizing three new 7-digit passwords and attempting to recall them after reading eighteen 7-digit numbers out loud is obviously a difficult one, and our results reflect this fact. Using a simple binary (right/wrong) metric, the Overt-Guidance condition posted the highest number of correctly recalled passwords with an average accuracy of $36 \%$. The least accurate was the No-Image condition, with an average accuracy of only $10 \%$. The average accuracy of the Image-Only and Subtle-Guidance conditions were both $18 \%$.

To obtain a more fine-grained accuracy measure, we then consider the average number of password digits recalled correctly and the average number of correct but misplaced digits.

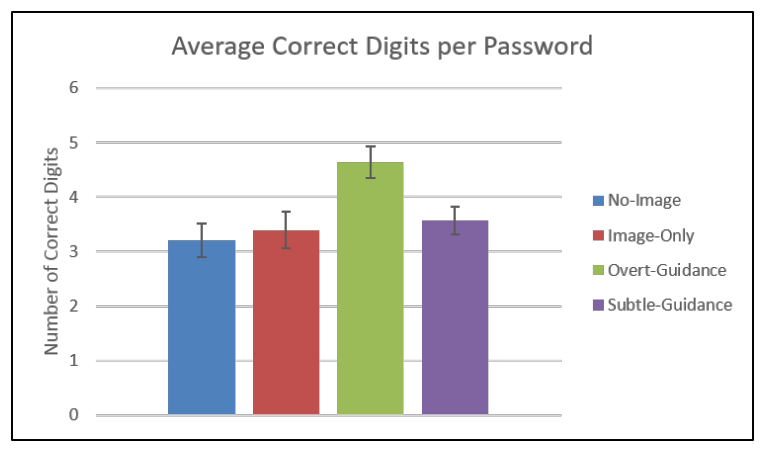

Figure 2: Average number of digits correctly recalled for each condition. Error bar indicates standard error.

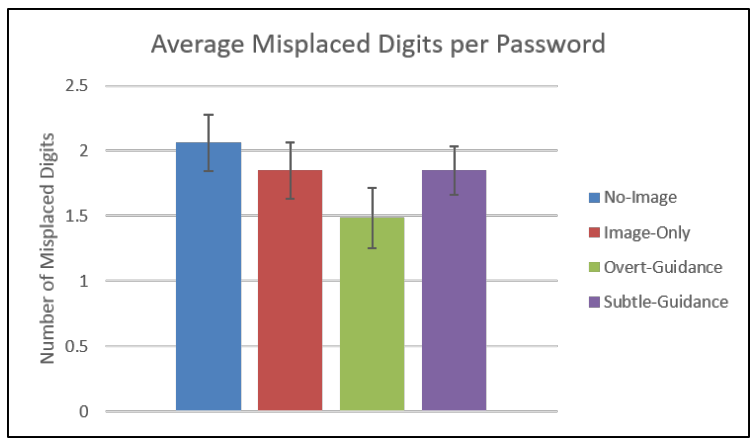

Figure 3: Average number correct but misplaced digits. Error bar indicates standard error.

Figure 2 shows the average number of password digits recalled correctly. As expected this accuracy measure during the OvertGuidance condition is significantly higher than the others. While not significant, the Subtle-Guidance condition also resulted in a higher average number of correct digits per password than the Image-Only and No-Image conditions. Figure 3 shows the average number of correct but misplaced digits. To prevent data from being skewed we only count a misplaced digit once. This helps to minimize the effect of random guesses leading to a more accurate result. The Overt-Guidance condition posted significantly lower misplaced digits compared to the other conditions. The NoImage condition posted a significantly higher number of misplaced digits. A single factor ANOVA was conducted between subjects to compare the four conditions. The results from the ANOVA indicated that these conditions were significantly different with $p$ values $<0.05$. These observations were further analyzed using independent-sample t-tests which are summarized in Table 1.

\section{Table 1: Significant T-test Results}

\begin{tabular}{|c|c|c|}
\hline Test Performed & $t$ value & $p$ value \\
\hline Correct Digits; No-Image vs. Overt & 3.33 & $<0.05$ \\
\hline Correct Digits; Image-Only vs. Overt & 2.78 & $<0.05$ \\
\hline Correct Digits; Subtle vs. Overt & 2.52 & $<0.05$ \\
\hline Misplaced; No-Image vs. Image-Only & 1.61 & $<0.05$ \\
\hline Misplaced; No-Image vs. Subtle & 1.61 & $<0.05$ \\
\hline Response Time; No-Image vs. Overt & 2.21 & $<0.05$ \\
\hline Response Time; No-Image vs. Subtle & 2.43 & $<0.05$ \\
\hline Response Time; Image-Only vs. Overt & 1.98 & $<0.05$ \\
\hline Response Time; Image-Only vs. Subtle & 1.98 & $<0.05$ \\
\hline Dwell-Time; Subtle vs. Overt & 1.19 & $<0.05$ \\
\hline
\end{tabular}




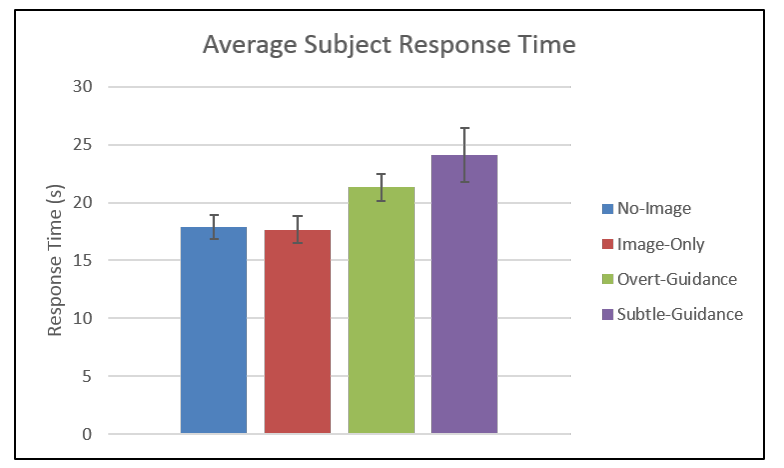

Figure 4: Average time used to recall password for each condition. Error bar indicates standard error.

\subsection{Response Time}

Response time was measured from the onset of the system beep until the password entry was completed. The average response time across all conditions is shown in Figure 4. The average response time for the gaze guided conditions were significantly slower than that of the unguided conditions (see Table 1). One possible explanation for this could be the additional cognitive load of having to follow the visual cues on screen.

\subsection{Dwell-time on Target Regions}

Dwell-time is the duration for which a subject fixates on an area of interest (AOI). For the gaze guided conditions, we define AOIs to be within $3^{\circ}$ of visual angle of the regions marked by the subject. We observed no significant difference in average dwell-time between the gaze guided conditions. Figure 5 shows example scanpaths and marked regions from one subject for the gaze guided conditions.

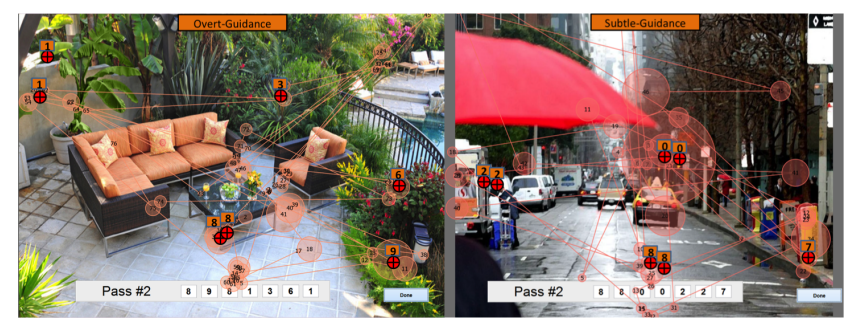

Figure 5: Example scanpaths from gaze guided conditions. The marked regions are represented using a red circle with a black cross-hair, and a number above indicating the associated digit.

\section{Conclusion and Future Work}

We analyzed the effect of gaze guidance on password recollection. Results indicate that guiding attention aids in password recollection, with Overt-Guidance having significantly higher accuracy compared to the other conditions. Response time indicates that participants took more time to recall passwords when there was gaze guidance. Dwell-time in target regions for the gaze guided conditions were similar. For future work, we would like to perform another study where subjects associate regions in the image, but are not gaze guided. This will be used to establish a baseline for comparison with gaze guided conditions. It would also be interesting to observe participants performing this task over an extended period of time to see if they converge to some optimal strategy for establishing spatial associations.

\section{Acknowledgements}

This material is based on work supported be the National Science Foundation under Award No. IIS-0952631. Any opinions, findings, and conclusions or recommendations expressed in this material are those of the author(s) and do not necessarily reflect the views of the National Science Foundation.

\section{References}

Awh, E., Anllo-Vento, L., Hillyard, S., et al. 2000. The role of spatial selective attention in working memory for locations: evidence from event-related potentials. Cognitive Neuroscience, Journal of 12, 5, 840-847.

Bailey, R., McNamara, A., Sudarsanam, N., And Grimm, C. 2009. Subtle gaze direction. ACM Trans. Graph. 28, 4 (Sept.), 100:1-100:14.

Groen, M., And Noyes, J. 2010. Solving problems: How can guidance concerning task-relevancy be provided? Comput. Hum. Behav. 26 (November), 1318-1326.

Hutton, P. 1993. History as an Art of Memory. University of Vermont.

ITTI, L., AND Koch, C. 2000. A saliency-based search mechanism for overt and covert shifts of visual attention. Vision Research 40, 10-12 (May), 1489-1506.

JudD, T., Durand, F., AND Torralba, A. 2012. A benchmark of computational models of saliency to predict human fixations. In MIT Technical Report.

Lu, W., Duh, H.-L., Feiner, S., And Zhao, Q. 2014. Attributes of subtle cues for facilitating visual search in augmented reality. Visualization and Computer Graphics, IEEE Transactions on 20, 3 (March), 404-412.

McNamara, A., Bailey, R., And Grimm, C. 2009. Search task performance using subtle gaze direction with the presence of distractions. ACM Trans. Appl. Percept. 6 (Sept.), 17:1-17:19.

Miller, G. 1956. The magical number seven, plus or minus two: Some limits on our capacity for processing information. The Psychological Review 63, 81-97.

PriviterA, C. M. 2006. The scanpath theory: its definitions and later developments. In Electronic Imaging 2006, International Society for Optics and Photonics, 60570A-60570A.

QVArfordt, P., BIEhl, J. T., Golovchinsky, G., AND DUNNINGAN, T. 2010. Understanding the benefits of gaze enhanced visual search. In Proceeding of 2010 Symposium on EyeTracking Research \& Applications, ACM, NY, USA, 283-290.

Sridharan, S., Bailey, R., McNamara, A., And Grimm, C. 2012. Subtle gaze manipulation for improved mammography training. In Proceedings of the Symposium on Eye Tracking Research and Applications, ACM, NY, USA, ETRA '12, 75-82.

Veas, E. E., Mendez, E., Feiner, S. K., And Schmalstieg, D. 2011. Directing attention and influencing memory with visual saliency modulation. In Proceedings of the SIGCHI Conference on Human Factors in Computing Systems, ACM, NY, USA, CHI '11, 1471-1480.

WOLFE, J. M. 1994. Guided search 2.0 a revised model of visual search. Psychonomic bulletin \& review 1, 2, 202-238. 